\title{
L'attribution des classes aux enseignants: le cas des écoles primaires
}

\author{
A Christine Leroy-Audouin, Bruno Suchaut \\ bruno.suchaut@u-bourgogne.fr \\ christine.leroy-audouin@u-bourgogne.fr
}

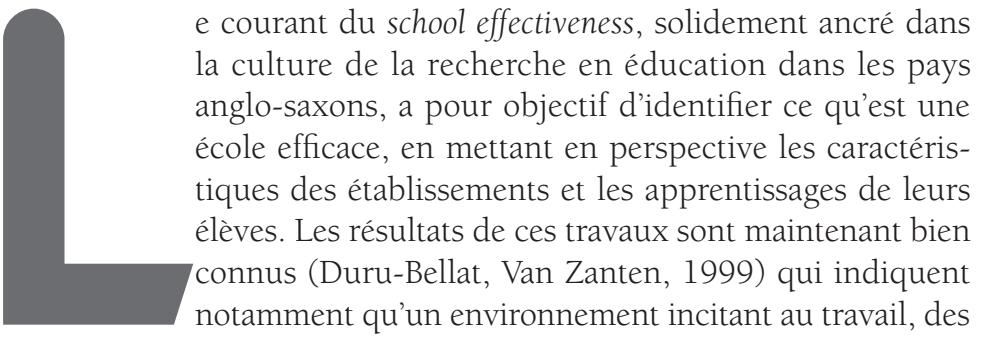
attentes élevées et positives vis-à-vis des élèves, des évaluations fréquentes, un climat discipliné sont autant d'éléments qui concourent aux progrès des élèves. Parmi ces constats, la notion de climat a donné lieu à de nombreux développements et il semble acquis que les caractéristiques de l'équipe pédagogique (stabilité, mobilisation, motivation, coopération...) et le type de « leadership » exercé par le chef d'établissement en sont des éléments importants (Meuret, 2003). Ces recherches s'appliquent également à l'enseignement primaire dans les pays anglo-saxons puisque les directeurs y disposent d'un statut hiérarchique. Elles montrent alors que la façon dont le directeur remplit sa fonction "managériale » au sein de l'équipe a un rôle crucial sur la vie de l'école et ses résultats (Bossert et al., 1982), notamment parce que son pouvoir de décision concerne à la fois le recrutement des enseignants et leur assignation à une classe dans laquelle ils exercent, contrairement au niveau secondaire, à temps complet (Goldhammer et Becker, 1971; Warren, 1975).

En France, les recherches sur cette thématique sont relativement récentes et concernent pour l'essentiel les collèges. Il a en effet fallu attendre que l'établissement soit reconnu comme une réalité organisationnelle et politique (Demailly, 2000) pour que se développe un questionnement sur les effets du contexte de scolarisation. Dans cette perspective, la sociologie des organisations a apporté un éclairage particulier à l'analyse de l'identité des 
établissements, qui finalement se révèle être « un montage composite entre des registres d'actions qui se réfèrent à des principes de légitimité différents et souvent antagonistes » (Derouet, 1987). Le rôle du chef d'établissement y est évidemment primordial, à qui il revient de faire construire un projet éducatif aux membres de l'équipe et de favoriser l'implication et la mobilisation de tous. Quand bien même ce rôle lui est clairement et institutionnellement dévolu dans l'enseignement secondaire, sa capacité d'action dans la gestion interne de l'établissement n'est, en réalité, que partielle et souvent difficile à mettre en place (Dupriez, 2003).

Que dire alors de ce qui se passe dans les écoles primaires? Les recherches sur ce niveau d'enseignement sont très rares. En effet, dans le contexte français, le directeur d'école primaire n'a aucun pouvoir hiérarchique dans l'établissement et, hormis les décharges horaires dont il peut éventuellement bénéficier, sa charge d'enseignement est identique à celle de ses collègues. Il n'est finalement qu'un enseignant parmi les autres et il ne peut en principe imposer une quelconque décision. Ce statut, manifestement difficile à assumer, a été l'objet ces dernières années de nombreuses revendications et de " grèves administratives », bloquant la remontée des informations réclamées par l'administration centrale. Si l'augmentation de la prime et l'élargissement des décharges de classe liées à la fonction sont clairement revendiquées par les syndicats, la question de la création d'un véritable corps des directeurs d'école reste en suspens, depuis longtemps d'ailleurs puisque dès 1987, la création d'un corps de " maîtres-directeurs » par René Monory avait engendré une levée de boucliers.

Les problèmes se posent pourtant de façon similaire aux deux niveaux d'enseignement. Ecoles, collèges et lycées partagent en effet des cadres structurels porteurs de concepts communs: le premier concerne les acteurs et « suppose que les systèmes sont agis de l'intérieur par des agents, et donc susceptibles d'être transformés », le second concerne l'autonomie et postule « qu'il existe des marges de manouvre laissées à la disposition des établissements » (Cousin, 2000). Enfin, et quel que soit le niveau, l'autorité instituée n'est en aucun cas suffisante et c'est la légitimité reconnue au chef d'établissement qui importe dans le style de leadership adopté (Cousin, 1998) et dans la nature et l'efficacité des interactions au sein de l'équipe.

Cet article, issu d'une recherche plus large consacrée aux modes de groupements des élèves (Leroy-Audouin, Suchaut, 2005), se focalise précisément sur le niveau primaire et, dans l'objectif d'identifier les dynamiques relationnelles internes, il se base sur une décision essentielle dans le fonctionnement des écoles: celle de l'assignation des enseignants à leur classe, ou plutôt du choix des enseignants de leur classe. La façon dont se prend cette décision est d'autant plus intéressante à analyser que vont se confronter, en l'absence d'arbitrage institutionnel et hiérarchique, des intérêts individuels, souvent concurrents car identiques.

Des entretiens semi-directifs, d'une durée d'une heure trente environ, ont été conduits auprès de 18 directeurs d'école, interrogés entre autre, sur la façon dont se déroulent dans leur école les procédures relatives au choix des enseignants de 
leur classe. Leur ancienneté moyenne dans la fonction s'élève à 7 ans et le nombre d'écoles dans lesquelles cette fonction a été exercée est faible en général (moins de 2 écoles). Par conséquent, l'ancienneté sur le poste actuel est en moyenne relativement longue (5 ans). La majorité des ces directeurs (directrices) a la responsabilité d'une classe (seuls trois d'entre eux sont totalement « déchargés »), dans plus de $80 \%$ des cas à hauteur de 3/4 de temps, ce qui correspond à environ une journée de « décharge» par semaine.

Par ailleurs, des données relatives à la structure des classes dans ces écoles ont été collectées. Il s'agit d'abord du nombre total d'élèves et de leur répartition par niveau, du nombre de classes constituées, de leur effectif et de leur type (simple ou multiple), et enfin, pour chacune des classes dans chaque école, des caractéristiques des 141 enseignants concernés (âge, sexe, ancienneté générale et dans l'école, poste occupé précédemment).

\section{Le consensus: refus du cours multiple et priorité aux intérêts individuels}

Le point commun à toutes les écoles est qu'au-delà du relatif isolement de chacun dans sa classe, les directeurs insistent sur la notion d'équipe; en est pour preuve le très grand nombre d'occurrences des termes « collègues », « équipe » et « consensus » qui apparaissent d'autant plus fréquemment dans les discours que les acteurs sont en présence depuis longtemps dans l'école:

« mais vous voyez, ce sont des gens qui travaillent ensemble depuis longtemps donc ça ne pose aucun problème » (école 3) ; « oui, c'est vraiment collégial, c'est-à-dire qu'il y a une espèce de confiance réciproque qui est établie depuis longtemps » (école 5).

Limportance de cette histoire commune est soulignée par Gather-Thurler (1994) qui montre que les organisations subissent le poids du passé qui s’inscrit dans la mémoire des membres et les unit. Se crée ainsi un fonds culturel commun aux enseignants d'une école et celui-ci, d'autant plus riche que la stabilité de l'équipe est grande, véhicule « des solutions construites, héritées et partagées en son sein » (Gather-Thurler, 1994).

Cette cohésion apparente est particulièrement visible au niveau des priorités pédagogiques établies dans l'organisation de l'école. En fait, l'évitement des cours multiples reste l'argument fondateur de la constitution des classes (Leroy-Audouin, Suchaut, 2005) et il est partagé par l'ensemble des enseignants, quand bien même il pourrait engendrer des déséquilibres très grands au point de vue des effectifs d'élèves:

« alors en général, on évite un maximum les cours doubles et la politique est dans notre école: il vaut mieux une classe chargée que trop de cours doubles » (école 3).

Une raison principale justifie aux yeux des directeurs la place première donnée à ce critère dans la constitution des classes. Elle est strictement d'ordre pédago- 
gique et renvoie au surcroît de travail que les cours multiples occasionnent aux enseignants, en terme de préparation d'abord:

« en tant qu'enseignant, on accepte mal les cours doubles parce que ça fait quand même une double préparation. Ca fait du jonglage, pas continuel mais presque entre les deux niveaux » (école 4); « c'est beaucoup de travail pour l'enseignant, c'est ça le problème » (école 13).

La conduite de la classe au quotidien est également jugée plus difficile dans les cours multiples:

« un enseignant en cours double fait la girouette toute la journée. C'est un one-man-show, quoi! » (école 16); " physiquement, il fallait que je me mette comme ça face aux CM2 pour leur parler et je parlais aux CM1. C'était affreux, j'étais constamment insatisfait. Il m'avait manqué de temps pour ceux-là, j'avais donné trop de temps aux autres » (école 14).

Si l'évitement des cours multiples est la règle dans la constitution des classes, il n'en reste pas moins que la France est, selon une étude réalisée dans le cadre de l'OCDE (M.E.N., 1997), l'un des pays dans lesquels, en raison de la petite taille des écoles, les classes à cours multiples sont les plus fréquentes: elles concernent environ 80 \% des écoles élémentaires. Le consensus très fort observé précédemment sur le rejet des cours multiples peut voler en éclat alors et conduire à l'émergence de situations potentiellement conflictuelles quand les enseignants doivent choisir leur classe, notamment si un cours multiple se présente. C'est la raison pour laquelle, un directeur souligne qu'au moment de la constitution des classes, on songe déjà aux individus:

« il n'y a pas encore trop d'enjeu personnel, quoique ça commence : où je vais me mettre?.... mais attention, là j'avais un CE2, là il va en manquer un...» (école 5).

Si les enseignants se rassemblent massivement autour de certaines valeurs, par conséquent, une certaine rivalité les oppose aussi et l'esprit d'équipe évoqué précédemment doit respecter, voire servir, les intérêts individuels en présence. Il s'agit donc de trouver un compromis et d'apporter une réponse pratique à ce problème, par la mise en place de négociations entre des acteurs cherchant à préserver leur propre intérêt. Dans cette perspective, Maroy et Dupriez (2000) évoquent la notion de « régulation »: celle-ci se comprend comme un processus social de production de règles du jeu, prenant place dans un espace daté et localisé. Cette notion accorde, conformément à d'autres travaux d'ailleurs (Reynaud, 1993; Friedberg, 1993), un poids central à l'histoire de l'équipe, qui favorise (ou freine parfois) la production, l'appropriation, voire le remodelage de ces règles. Les déclarations des directeurs révèlent ainsi qu'il existe à la fois des règles valables dans l'ensemble des écoles qui s'appliquent à tous les enseignants, et des arrangements locaux, liés à l'équipe et à l'histoire de chaque école. 


\section{Des usages faisant force de règles}

Selon le calendrier rapporté par les directeurs interrogés, après avoir décidé des classes à constituer pour la rentrée suivante, il s'agit ensuite de les attribuer aux enseignants. Ce n'est que dans une dernière étape qu'y seront affectés les élèves. C'est donc en fonction d'une classe considérée dans sa structure (type de cours et niveau) et pas dans sa composition (le groupe d'élèves) que les enseignants doivent se prononcer. Concernant le type de classe, cela a été dit précédemment, la préférence va nettement aux cours simples. Concernant le niveau d'enseignement, s'il apparaît en moyenne une certaine spécialisation des enseignants en école maternelle ou en école élémentaire, en revanche, la question des différents niveaux élémentaires est rarement abordée tant elle semble liée à ce qui semble être une sensibilité personnelle. Selon les directeurs en effet, il n'existe pas

«le sentiment que ce soit plus difficile dans un niveau que dans un autre » (école 8) et en fait, «c'est très variable selon les personnes » (école 1), « c'est chacun... chacun selon son caractère » (école 14).

Si tous les niveaux de classe n'exercent pas le même attrait sur les enseignants, on peut imaginer alors que ces derniers tentent, dans la mesure du possible, de conserver d'une année sur l'autre «leur » classe. Les déclarations des directeurs indiquent que c'est effectivement le cas dans la majorité des écoles.

\section{L'attachement à " sa " classe}

Lattachement des enseignants à un même niveau scolaire est une réalité d'autant plus forte que l'organisation n'est pas remise en cause d'une année sur l'autre, comme c'est le cas dans les écoles suivantes:

« les gens qui avaient ces classes-là cette année reprendront les mêmes » (école 2); « pour l’instant, on garde nos classes » (école 12).

Par ailleurs, cette tendance se confirme quand les enseignants sont plus âgés ou plus anciens dans le niveau:

" je crois qu'il y a aussi des règles un peu implicites: le collègue qui a des CM2 depuis 15 ans, on chamboule pas tout, on va pas lui donner un $\mathrm{CP}$ » (école 5); « c'est des gens qui sont là depuis un certain temps, qui ont leurs habitudes de cours, de niveau » (école 18).

Cette « spécialisation », qui correspond effectivement aux préférences des enseignants pour une classe d'âge particulière, renvoie donc également à un certain confort dans l'exercice du métier:

« il y a d'autres collègues qui vont me dire: «attends, tu ne vas pas me donner un cours double alors que je pars en retraite l'année prochaine? » C'est rarement des arguments pédagogiques mais je me mets à leur place aussi... c'est pas marrant » (école 2). 
Cette spécialisation n'est évoquée de façon positive et constructive que par deux directeurs qui y voient, au contraire, l'opportunité d'un approfondissement:

« le collègue qui fait du $\mathrm{CP}$, moi je souhaite qu'il reste sur le cycle 2 puisque c'est quelqu'un qui s'est énormément investi sur l'apprentissage de la lecture, qui fait un excellent travail, d'abord de pédagogue et dont on a énormément besoin au niveau de la formation IUFM » (école 1).

Lanalyse des données concernant les classes et les enseignants de ces dix-huit écoles confirme cette tendance et le tableau suivant rend compte des mouvements d'une année sur l'autre pour tous les niveaux d'enseignement.

Tableau I: Niveau d'enseignement de l'année précédente et de l'année en cours (enseignants ayant en charge des cours simples)

\begin{tabular}{|c|c|c|c|c|c|c|c|c|c|c|c|}
\hline \multirow{3}{*}{ 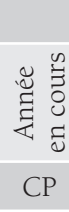 } & \multicolumn{11}{|c|}{ Année précédente } \\
\hline & \multicolumn{2}{|r|}{$\mathrm{CP}$} & \multicolumn{2}{|c|}{ CE1 } & \multicolumn{2}{|c|}{ CE2 } & \multicolumn{2}{|c|}{$\mathrm{CMl}$} & \multicolumn{2}{|c|}{$\mathrm{CM} 2$} & \multirow{2}{*}{$\begin{array}{c}\text { Total } \\
20(100)\end{array}$} \\
\hline & 16 & $80 \%$ & 3 & $15 \%$ & 1 & $5 \%$ & 0 & 0 & 0 & 0 & \\
\hline CEl & 2 & $14,3 \%$ & 11 & $78,6 \%$ & 1 & $7,1 \%$ & 0 & 0 & 0 & 0 & $14(100)$ \\
\hline CE2 & 0 & 0 & 2 & $11,8 \%$ & 13 & $76,5 \%$ & 2 & $11,8 \%$ & 0 & 0 & $17(100)$ \\
\hline $\mathrm{CMl}$ & 1 & $6,3 \%$ & 0 & 0 & 1 & $6,3 \%$ & 11 & $68,8 \%$ & 3 & $18,8 \%$ & $16(100)$ \\
\hline $\mathrm{CM} 2$ & 0 & 0 & 1 & $4,5 \%$ & 0 & 0 & 5 & $22,7 \%$ & 16 & $72,7 \%$ & $22(100)$ \\
\hline
\end{tabular}

Les enseignants qui étaient en cours simple et qui le sont restés sont au nombre de 89. Parmi eux, les trois-quarts (75,3\%) ont strictement conservé leur niveau de classe, la « spécialisation » étant particulièrement marquée sur la classe de CP. On observe par ailleurs que les mouvements se réalisent en général au sein d'un même cycle d'enseignement: si le CE2, qui appartient pourtant au cycle 3, a une position très intermédiaire à cet égard, les « glissements » des enseignants sur le niveau précédent ou suivant sont extrêmement fréquents entre le CP et le CE1, et le CM1 et le CM2.

\section{Priorité aux plus anciens dans l'école}

En cas de difficulté ou de désaccord dans l'équipe, une règle univoque et commune à toutes les écoles dont le directeur a été interrogé, s'impose avec autorité: c'est celle de l'ancienneté dans l'école, qui détermine l'ordre dans lequel les enseignants vont s'exprimer:

« la coutume veut que ce soit les plus anciens dans l'école qui parlent en premier » (école 6) et «le dernier arrivé prend ce qu'on lui donne » (école 1). 
Les directeurs évoquent cette pratique en termes de « coutume » ou de « tradition » qui n'est que rarement remise en cause, y compris par les Inspecteurs de l'Éducation nationale. Dans toutes les écoles, cette démarche est la même, qui accorde aux plus anciens dans l'école le droit de choisir en premier alors que les nouveaux arrivants, même ceux dont l'ancienneté générale serait plus élevée, se doivent d'accepter ce qui « reste ».

Une exception à cette règle est parfois admise et le premier enseignant par l'ancienneté dans l'école peut être devancé par le directeur:

« en théorie, c'est le directeur qui choisit, ensuite les adjoints se répartissent les classes suivant leur ancienneté dans l'école » (école 15); « la tradition veut ça... la personne la plus ancienne choisit. En fait, le directeur choisit en premier puisque c'est par ordre de...» (école 7).

Certains directeurs utilisent cette prérogative tandis que d'autres la refusent, témoignant en cela de la façon dont ils gèrent la question de leur autorité au sein de l'équipe:

« le directeur, par sa fonction, a le choix... mais je n'aime pas imposer » (école 17); « ça veut dire que je n'utilise pas ma prérogative, normalement si je voulais, je pourrais choisir ma classe en premier et puis bon... comme je voudrais que justement on n'en arrive pas à se répartir les classes par ancienneté.... (école 8).

Par ailleurs, certains contournements de cette règle sont tolérés dans des situations particulières, et notamment en ce qui concerne la classe de CP qui ne doit pas être attribuée à un enseignant débutant ou un enseignant à temps partiel. On peut alors déroger à la règle de l'ancienneté dans l'école, pour éviter que le CP soit la classe qui « reste » à un nouvel arrivant sortant de formation.

Ladage selon lequel « les derniers arrivés sont les derniers servis » (école 17) se vérifie dans cet échantillon, ainsi que l'illustre le tableau 2 suivant, qui met en relation l'ancienneté des enseignants et le type de classe auquel ils sont affectés. Globalement et conformément aux déclarations des directeurs, il n'existe pas de différence selon l'ancienneté générale des enseignants puisque la règle concerne le dernier arrivé dans l'école. Celui-ci peut avoir un certain nombre d'années d'ancienneté dans l'école si cette dernière connaît peu de mouvements et il sera en général considéré, jusqu'à nouvel ordre, comme le moins ancien et par conséquent, susceptible de conserver une classe qu'il ne souhaitait pas: " et bien voilà, donc il y en a qui peuvent rester avec des cours doubles pendant des années » (école 1). Un indicateur a donc été créé, qui rend compte de l'identification dans chaque école de cet enseignant particulier ${ }^{1}$.

1. Dans quelques écoles, deux enseignants arrivés la même année sont considérés comme les derniers arrivés. 


\section{Tableau 2. - Type de classe occupé selon que l'enseignant est le dernier arrivé ou pas dans l'école ${ }^{2}$}

\begin{tabular}{|c|c|c|c|c|c|}
\hline Enseignants & \multicolumn{2}{|c|}{ Cours simple } & \multicolumn{2}{|c|}{ Cours multiple } & Total \\
\hline les plus anciens & 95 & $86,4 \%$ & 15 & $13,6 \%$ & $110(100)$ \\
\hline les derniers arrivés & 19 & $70,4 \%$ & 8 & $29,6 \%$ & $27(100)$ \\
\hline
\end{tabular}

Beaucoup plus fréquemment que les autres, les derniers arrivés se voient attribuer un cours multiple ${ }^{3}$, ce qui est confirmé par les directeurs:

«alors, c'est très embêtant les cours doubles parce que justement, les instits ne les prennent pas, donc c'est le dernier qui arrive qui, en général, se prend le cours double » (école 14); « et l'autre personne nouvelle, elle a pris ce qui restait: le cours double; ce n'était pas un cadeau empoisonné, on le sait maintenant, mais en septembre, on pouvait penser autrement » (école 17).

Globalement, on observe donc que l'attribution des classes selon ce critère d'ancienneté n'est pas optimale, dans la mesure où on confie à des enseignants débutants des classes plus complexes à gérer sur le plan pédagogique. On peut rapprocher ce constat des modes d'affectations dans les écoles, collèges et lycées, qui voient en général les enseignants les moins expérimentés nommés dans les établissements réputés les plus difficiles, en terme social notamment.

Au-delà de ces deux règles qui valent dans toutes les écoles, certains arrangements sont néanmoins consentis. Ils prennent en compte le contexte particulier de chaque école et l'histoire, voire le passif, de l'équipe, mais s'inscrivent en définitive et à leur tour, sous la férule de l'usage.

\section{Quelques arrangements...}

Deux grandes tendances se dégagent des discours des directeurs. La première a trait aux concessions qui sont faites aux enseignants qui se « dévouent» en acceptant les classes les moins prisées parce que «si on facilite le travail du maître, on facilite l'ambiance de la classe » (école 4). C'est notamment le cas des cours multiples:

« c'est une garantie d'avoir un peu moins d'élèves; ça compense la surcharge de boulot... enfin, ça équilibre » (école 18); « sur un cours double, c'est certain que de toute façon, c'est plus de boulot qu'un cours simple. Mais enfin, on essaie d'arranger l'emploi du temps de la personne, les effectifs, les services pour que, d'un autre côté, il s'y retrouve un petit peu » (école 2).

Au-delà de leur nombre, les élèves dont le maître aura la charge peuvent être objet de négociations également:

2. L'effectif de 137 enseignants diffère ici légèrement de celui annoncé auparavant du fait de données manquantes pour 4 enseignants.

3. Une analyse limitée aux seules écoles qui comptent des cours doubles accentue encore cet écart. 
« Mme X., cette maîtresse ayant un cours double, nous concevons tous que la prise d'un cours double implique certaines difficultés et certaines tâches supplémentaires. Donc, on va tous dans l'idée de faciliter le travail du maître, on lui accorde le choix de garder ou non ses élèves » (école 4); « la maîtresse qui va accueillir un cours double, en général, on lui met que des bons élèves » (école 14).

La seconde tendance, elle aussi citée par l'ensemble des directeurs, a trait à la mémoire du groupe et à la reconnaissance collective des renoncements individuels. Implicitement, s'instaure un roulement dans l'école, qui fait que chacun devra un jour ou l'autre se dévouer pour prendre la classe dont personne ne veut:

« le collègue qui fait une grosse concession, l'année suivante devient prioritaire. Ça, c'est la mémoire du groupe; c'est pas écrit mais on le sait [...] ça, ça fonctionne bien, les gens ont cette sorte de reconnaissance » (école 5); « en général, ça s’organise, ça va. Il y en a toujours un qui dit: « bon, c'est bon, je le prends... mais l'an prochain, si... est-ce que tu acceptes de le prendre? » (école 14); « par exemple, quelqu'un qui a été en cours double, on essaie, l'année d'après, de le remettre en cours simple... enfin d'alterner » (école 6).

Evidemment, si l'enseignant n'a pas honoré son engagement, alors son « dédommagement » est moins assuré, comme dans l'école 14 où « c'est celle qui a deux ans d'ancienneté qui a le cours double... si ça se produit encore l'année prochaine, comme elle a bénéficié de son congé maternité, c'est encore elle qui l'aurait je pense. Les collègues diraient: «attends, attends, t'as pas été beaucoup là l'an dernier $»$.

En fait, c'est toute la dialectique de l'articulation entre l'équipe et l'individu que résument ces discussions autour de l'attribution des classes aux enseignants. La conjugaison des différentes règles qui coexistent dans les écoles peut s'avérer extrêmement complexe à mettre en œuvre, même si le poids de l'ancienneté est prédominant. Il revient alors au directeur d'initier et piloter les débats et de faire parvenir son équipe à un accord, sans avoir le pouvoir d'imposer une solution. La tâche s'avère donc délicate et mieux connaître le positionnement du directeur au sein de l'équipe permet d'identifier les voies d'accès au consensus.

Les recherches montrent à cet égard que l'influence que peut avoir un directeur sur ses collègues dépend de son pouvoir et de son autorité (Bossert et al., 1982). Selon Swidler (1979), l'autorité représente le pouvoir investi dans une personne à qui le droit de décider a été reconnu: on peut donc avoir le pouvoir sans l'autorité, et c'est ce que tendent à montrer d'ailleurs les travaux concernant les chefs d'établissement du secondaire. Les directeurs n'ayant pas de pouvoir institutionnel en France, ils doivent « gagner » la reconnaissance de leur autorité au cours des discussions et négociations avec leurs collègues, en conduisant avec succès un certain nombre d'opérations délicates (conflits, répartition des classes, gestion des ressources...). Finalement, plus leur autorité est reconnue, plus leur pouvoir est grand et plus leur influence au sein de l'école est importante. 


\section{Comment le directeur établit-il et exerce-t-il son leadership?}

Différents positionnements ont été relatés au cours des entretiens, qui se caractérisent selon leur plus ou moins grande directivité vis-à-vis des autres enseignants, la position intermédiaire étant celle qui, sans surprise, est le plus souvent adoptée.

À un extrême se situent les directeurs les plus prescriptifs, qui évoquent un fonctionnement très hiérarchisé de l'équipe. Un tiers d'entre eux a cité ce type de relations, qui tend à asseoir l'autorité dans des rapports de force. Cela dit, il est intéressant de constater que cinq de ces six directeurs (directrices) le font pour narrer des pratiques qui ne sont pas les leurs ou alors exceptionnellement:

« je donne un avis... et je peux trancher mais c'est très exceptionnel... Sinon on va au clash » (école 1); « bon je sais que ma collègue précédente, ses dernières années de carrière, préférait organiser, enfin faire sa répartition de classes avec l'inspectrice, sans se préoccuper du choix des enseignants... ça... je trouve ça gênant quand même » (école 12).

Seuls deux directeurs revendiquent un positionnement intransigeant, qui selon eux, s'avère positif. On notera avec intérêt que ces deux directeurs viennent des deux plus grandes écoles de l'échantillon (respectivement 14 et 15 classes) et n'ont pas de charge d'enseignement:

« Ça m'est arrivé une année, c'est l'arbitraire. Bon, je suis le directeur, on n'arrive pas à se mettre d'accord, j'assume mon autorité et c'est comme ça puisqu'on n'arrive pas à se mettre d'accord. Alors, bon, en général, on évite... les gens ont vu que j'étais capable de le faire donc ils savent » (école 5); « autant quand je suis arrivé, pendant quelques années, j’ai insisté sur mon rôle de directeur en étant très ferme mais maintenant les choses sont en place, les gens me connaissent. Ils savent que c'est 8 h 30 et pas 31, que la récré dure 20 minutes et pas $21 \ldots$ mais maintenant j'essaie de laisser les choses tourner et les gens me disent qu'autant au début, je paraissais très directif et je déclenchais de l'animosité, autant maintenant les gens reconnaissent que c'était une bonne méthode parce qu'ils se sentent encadrés par des règles et un système qui est installé... et qui protège » (école 14).

Au-delà de ce type de positionnement, marginal et sans doute lié au contexte scolaire, à la taille de l'équipe et l'arrivée récente du directeur notamment, ce sont des rapports plus nuancés et conciliateurs que les directeurs évoquent le plus fréquemment. Les trois-quarts d'entre eux ont effectivement insisté sur les échanges, les discussions et les débats dans l'équipe, qui trouvent toujours ou presque une issue dans le consensus. Cela dit, une nuance peut être apportée à ce type de discours selon qu'est utilisé dans l'entretien le « je » (par opposition aux « eux ») qui témoigne d'une certaine distance, voire de hauteur par rapport aux enseignants et le «nous» ou « on » qui, au contraire, inclut le directeur dans l'équipe et rend compte alors à la fois de l'équivalence des statuts et d'une conciliation plus participative. Le premier cas, le moins fréquent, s'observe dans l'école suivante dans laquelle le directeur déclare: 
« moi je les laisse d'abord se chamailler, je les laisse d'abord évacuer les choses; ça fait du bien de se dire ce qu'on a envie de dire ou qu'on garde sur le cœur... et puis en général, ça s'arrange bien comme ça; sinon, quand je sens qu'il y a trop de torsions, il y a des critères... des critères administratifs».

Dans le second cas, le fait que les enseignants s' « entendent» bien, au sens littéral comme au sens figuré, est toujours évoqué par les directeurs, qui mentionnent en outre souvent des affinités personnelles:

" ça se passe bien, on est une école où on s'entend bien; dans les écoles où on s'entend moins bien, c'est beaucoup plus difficile » (école 1); « je crois que, oui heu..., c'est vraiment collégial, c'est-à-dire qu'il y a une espèce de confiance réciproque à la parole de l'autre et donc, il y a un enjeu important, mais ça se passe plutôt bien » (école 5).

Enfin, à l'autre extrémité du spectre, un dernier type de fonctionnement a été relaté, dans lequel le directeur peut aller jusqu'à s' « effacer » afin de préserver la cohésion de l'équipe. On notera que ce positionnement correspond plus à des situations ponctuelles qu'à un mode relationnel routinier, même s'il est parfois systématique lors de l'attribution des classes. C'est le cas de la directrice suivante:

«bon, moi... pour faciliter les choses et parce que je veux que l'équipe pédagogique soit quand même soudée, qu'il n'y ait pas trop de problèmes dans l'équipe et tout..., généralement je prends la classe qui reste, dont personne ne veut [...] alors c'est vrai que ça me fait beaucoup de travail mais pour moi, c'est moins dur que s'il y a des tensions dans l'équipe, pour moi c'est moins dur à gérer » (école 8).

Les autres directeurs s'estiment eux « arrangeants » ou conciliants mais leurs concessions ne vont pas aussi loin:

« j'ai choisi en m'arrangeant pour que mon choix ne gêne pas trop les anciens; j'ai fait en sorte que mon choix respecte un peu tout le monde » (école 7); « si vous voulez, quelques fois... bon... moi, j'essaie d'être arrangeante, mais pas à mon détriment quand même... je vais pas porter toute la charge de l'école, tous les problèmes de l'école sur mes épaules » (école 12).

Les déclarations des directeurs au sujet de la classe dont ils ont la charge confirment ces positionnements: tous n'ont manifestement pas choisi leur classe dans la mesure où sont entrées en conflit deux règles citées précédemment, la priorité donnée à l'ancienneté dans l'école et celle accordée à la fonction de direction. Il apparaît ainsi que si le directeur nommé est un nouvel arrivant, alors la règle de l'ancienneté s'applique à lui comme aux autres et il peut arriver qu'il prenne la classe qui « reste ». C'est ce qui s'est explicitement produit pour 6 des 18 directeurs interrogés, et notamment ceux qui se sont vus attribuer les cours doubles. Ces directeurs nouvellement nommés se sont donc trouvés face à une surcharge de travail, administrative et pédagogique. C'est peut-être la raison pour laquelle il est si difficile actuellement de pourvoir les postes de direction dans les petites écoles: 
«... parce que le CM1-CM2, c'était la classe qui restait... dont personne ne voulait » (école 8); « disons que quand je suis arrivée, le poste qui restait était une classe de CM1-CM2 et donc je me suis adaptée à la classe et par la suite, bon... ben c'est vrai que j'y ai pris plaisir et personne ne souhaitait avoir cette classe » (école 12).

D’autres niveaux de classe, pourtant généralement moins redoutés, ne sont néanmoins pas ceux qu'auraient choisis personnellement certains directeurs:

« sur la commune, il existe des classes de neige et tous les enfants des classes de CMI partent 13 jours; il est très difficile de trouver un enseignant qui accepte [...] donc, contrainte et forcée, je fais la classe de CMl depuis 8 ans » (école 13); « j’ai un CEl, c'est ce qui restait » (école 16).

Finalement, la mise en perspective des deux types de discours, concernant d'une part les règles mobilisées pour l'attribution des classes et d'autre part, le mode de leadership du directeur, fait apparaître clairement qu'un directeur nouvel arrivant dans une école n'est en définitive qu'un enseignant parmi les autres, notamment s'il a une charge d'enseignement. Dans ce cas, il se soumet aux règles en vigueur pour le choix de sa classe en s'effaçant devant les plus anciens, et son autorité doit se construire dans le temps. En revanche, s'il est déchargé, il peut se permettre d'agir avec plus d'autorité, voire de « passer en force », ainsi qu'en ont témoigné précédemment deux directeurs qui reconnaissent dans le même temps avoir suscité une certaine hostilité à leur égard.

On retrouve ici la notion de consensus, telle qu'elle est développée par Francfort, Sainsaulieu et al. (Draelants, 2002), qui montrent que le consensus renvoie, d'une part à un phénomène de partage de valeurs et d'orientations relatives à l'organisation et, d'autre part, à un système de relations sociales au sein du groupe qui a pour objectif d'éviter les conflits interindividuels. Le système de négociations se caractérise alors par le fait qu'il n'y a pas d'acteur clé exerçant sa suprématie sur les autres. La plupart des acteurs s'engagent sur des problèmes de coopération ponctuels, mènent des négociations et réalisent des ajustements dans un espace assez souple et peu formalisé : ce « compromis négocié » (Dupriez, 2003) ou working consensus (Bossert et al., 1982) s'appuie principalement sur des éléments pragmatiques et concrets. À cet effet, l'application de quelques règles, simples et univoques, comme celles qui ont été évoquées, augmente la prédictibilité des décisions, réduisant ainsi les ambiguités et les malentendus (Fuller et al., 1982), et par conséquent les conflits.

Les déclarations des directeurs interrogés dans cette recherche valorisent en effet le maintien d'un climat pacifique et convivial dans l'école plus qu'un objectif d'efficacité pédagogique dans l'appariement entre enseignants et classes. On rejoint à cet égard un axe de lecture tracé par Blake et Mouton (cités par Bossert et al., 1982), qui distinguent, dans les discussions au sein des équipes, les considérations relatives aux collègues (affective leadership) de celles orientées vers les résultats (instrumental leadership). 


\section{Conclusion}

De toutes les typologies réalisées sur la connaissance des modes relationnels entre enseignants dans les écoles (travaux cités par Gather-Thurler, 1994), on peut sans doute en retenir une qui semble bien témoigner de la réalité rapportée par les directeurs interrogés. Il s'agit de la « grande famille » qui caractérise un mode relationnel à l'intérieur duquel les membres de l'équipe sont parvenus à une certaine forme de paix sociale, grâce à la soumission de chacun à un jeu de règles explicites et implicites. Dans ce type de relations, c'est le bien-être des uns et des autres qui importe et la sociabilité prime dans la recherche de solutions pragmatiques au cours de laquelle le directeur joue le rôle de médiateur (Gather-Thurler, 1994).

Si les auteurs précédents comparent cette « grande famille» à une «fanfare de village », le consensus, tel qu'il est décrit par les directeurs interrogés, pourrait n'être néanmoins qu'un paravent de la rivalité des désirs et intérêts individuels de chacun. On peut déplorer à cet égard de ne pas disposer dans cette recherche de l'avis des enseignants sur le sujet. En effet, il est possible que certains directeurs aient eu tendance à valoriser des pratiques positives plutôt que d'évoquer les conflits interpersonnels au sein de l'école et les difficultés, voire les échecs, à les résoudre.

Les travaux anglo-saxons montrent que les directeurs d'écoles usent en général seuls de leur pouvoir de décision pour affecter les enseignants dans les classes mais que la prise en compte des préférences de ces derniers (voire des parents) constitue une variable d'ajustement permettant de préserver la stabilité de l'équipe et la collégialité (Heck et al., 1989). C'est ainsi que ce type de décisions et la façon dont elles sont prises au quotidien sont centraux et témoignent des compétences des directeurs (Glasman et Heck, 1987) : l'efficacité des chefs d'établissement pourrait alors transiter par cet élément organisationnel, en influençant non seulement le climat de l'école, mais aussi les progressions des élèves (Monk, 1992). Cette hypothèse est relayée empiriquement par un certain nombre de recherches (citées par Bossert et al., 1982), qui montrent que le niveau de satisfaction des enseignants est lié, certes indirectement, aux performances de leurs élèves (Lee et al., 1991).

Finalement, ces observations interrogent la question de l'attribution des classes aux enseignants dont les principes généraux semblent reposer sur une régulation centrée sur l'habitude et qui donne la priorité aux intérêts individuels et à l'ancienneté. Lintérêt général et celui des élèves ne constituent donc pas une priorité, alors même que les recherches ont montré que l'influence des enseignants sur les progressions des élèves était très forte (Bressoux, 1994). On peut se demander alors s'il est possible de réaliser des appariements entre maîtres et classes (envisagés sous l'angle de la composition des groupes d'élèves) optimaux dans le même contexte institutionnel ou s'il est nécessaire de faire évoluer le statut des directeurs. À l'heure actuelle, la primauté de l'ancienneté et de l'habitude constitue manifestement un frein au changement organisationnel et pédagogique.

Christine Leroy-Audouin, Bruno Suchaut, IREDU-université de Bourgogne. 


\section{Biblographie}

Bossert S.T., Dwyer D.C., Rowan B., Lee G.V. (1982), The Instructional Management Role of the Principal, Educational Administration Quaterly, 18 (3), p. 34-64.

Bressoux P. (1994), Les recherches sur les effets-écoles et les effets-maîtres. Revue française de pédagogie, $\mathrm{n}^{\circ} 108,91-137$.

Cousin O. (1998), L'efficacité des collèges. Sociologie de l'effet établissement, Paris: PUF.

Cousin O. (2000), Politiques et effets-établissements dans l'enseignement secondaire, in Van Zanten A. (sous la dir. de), L'école, l'état des savoirs, ed. La découverte, p. 139-148.

Demailly L. (2000), Management et évaluation des établissements, in Van Zanten A. (sous la dir. de), L'école, l'état des savoirs, ed. La découverte, p. 130-138.

Derouet J.-L. (1987), Contrat et établissement scolaire, in Burguière et al., Contrat et éducation. La pédagogie du contrat, le contrat en éducation, Paris: L'Harmattan/INRP, coll. CRESAS nº 6.

Draelants H. (2002), Limpact des structures et de l'organisation d'un système scolaire sur la production d'inégalités en son sein, Esprit critique, Vol. 4, n 5 .

Dupriez V. (2003), De l'isolement des enseignants au travail en équipe: les différentes voies de construction de l'accord dans les établissements, Cahier de recherche du GIRSEF, n² 23, 20 p.

Duru-Bellat M., Van Zanten A. (1999), Sociologie de l'école, A. Colin, Paris.

Friedberg E. (1993), Le pouvoir et la règle, Paris: Seuil.

Fuller B., Wood K., Rapoport T., Dornbush S.M. (1982), The organizational context of individual efficacy, Review of Educational Research, 52 (1), p. 7-30.

Gather Thurler M. (1994), Relations professionnelles et culture des établissements scolaires : au-delà du culte de l'individualisme?, Revue française de pédagogie, n 109, p. 19-39.

Glasman N.S., HeCK R.H. (1987), Evaluation in decision making: the case of assigning teachers to classrooms, Administrator's Notebook, 32 (5), 1-4.

Goldhammer K., Becker G. (1971), Elementary school principals ans their schools, Eugene, Center for the Advanced Study of Educational Administration.

Heck R., Marcoulides G., Glasman N. (1989), The application of causal modeling techniques to administratives decision making: The case of teacher allocation. Educational Administration Quarterly, 25 , p. $253-267$.

Lee V.E., Dedrick R.F., Smith J.B. (1991), The effect of the social organization of schools on teachers'efficacy and satisfaction, Sociology of Education, 64, p. 190-208.

Leroy-Audouin C., Suchaut B. (2005), La constitution des classes dans les écoles: contraintes de contexte ou stratégies d'acteurs? Rapport intermédiaire pour le PIREF, 82 p.

Maroy C., Dupriez V. (2000), La régulation dans les systèmes scolaires, proposition théorique et analyse du cadre structurel en Belgique francophone, Revue française de pédagogie, n 130, p. 73-87.

MEN. (1997) Enquête internationale sur le fonctionnement des écoles élémentaires, Les dossiers d'Education et formations, $\mathrm{n}^{\circ} 85$.

Meuret D. (2003), Efficacité et équité des collèges. L'effet établissement. In Derouet J.-L. (sous la dir. de), Le collège unique en question, Paris: PUF.

Monk D. (1987), Assigning elementary pupils to their teachers, Elementary School Journal, 88, p. 167-187.

Monk D. (1992), Educational productivity research: An update and assessment of its role in education finance reform, Educational Evaluation and Policy Analysis, 14, p. 307-332.

Reynaud J.-D. (1993), Les règles du jeu. L'action collective et la régulation sociale, Paris: A. Colin.

Swidere A. (1979), Organization without authority: Dilemmas of Social Control in Free Schools, Cambridge: Harvard University Press.

WARREN R. (1975), Context and isolation: The teaching experience in the elementary school, Human Organization, 32 (2), p. 139-148. 